\title{
Prevalência e fatores associados ao abandono do tratamento da tuberculose
}

\author{
Prevalence and associated factors of tuberculosis treatment abandonment \\ Prevalencia y factores asociados al abandono del tratamiento de la tuberculosis
}

Como citar este artigo:

Sousa GJB, Maranhão TA, Leitão TMJS, Souza JT, Moreira TMM, Pereira MLD. Prevalence and associated factors of tuberculosis treatment abandonment. Rev Esc Enferm USP. 2021;55:e03767. https://doi.org/10.1590/S1980-220X2020039203767

\section{Deorge Jó Bezerra Sousa ${ }^{1}$ \\ Thatiana Araujo Maranhão \\ Terezinha do Menino Jesus Silva Leitão ${ }^{3}$ \\ Jerffeson Teixeira de Souza ${ }^{4}$ \\ Thereza Maria Magalhães Moreira ${ }^{1}$ \\ Maria Lúcia Duarte Pereira ${ }^{1}$ \\ ${ }^{1}$ Universidade Estadual do Ceará, Programa de Pós-graduação Cuidados Clínicos em Enfermagem e Saúde, Fortaleza, CE, Brasil. \\ ${ }^{2}$ Universidade Estadual do Piauí, Curso de Enfermagem, Parnaíba, PI, Brasil. \\ ${ }^{3}$ Universidade Federal do Ceará, Departamento de Saúde Comunitária, Fortaleza, CE, Brasil. \\ ${ }^{4}$ Universidade Estadual do Ceará, Departamento de Computação, Fortaleza, CE, Brasil.}

\begin{abstract}
Objective: To estimate the prevalence of tuberculosis treatment abandonment and its associated factors. Method: Cross-sectional study which used cases of tuberculosis in the System of Information on Notification Aggravations (Sistema de Informação de Agravos de Notificação) from 2001 to 2017 in Ceará state. This study included 74,006 cases and the outcome was the closing situation "treatment abandonment". A multivariate analysis was performed to estimate the association between the variables with abandonment. Results: Throughout the period, the abandonment rate was $12.54 \%$. A higher abandonment prevalence was verified among people who live in the urban zone $(\mathrm{PR}=2.45 ; 95 \% \mathrm{CI}$ : 2.20-2.74), who are readmitted after abandonment ( $\mathrm{PR}=2.84$; 95\%CI: 2.68-3.01), among those notified as recurrent $(\mathrm{PR}=1.22 ; 95 \% \mathrm{CI}: 1.10-1.35)$ and among drinkers $(\mathrm{PR}=1.50 ; 95 \% \mathrm{CI}: 1.42-1.58)$. Those who were sputum smear-positive $(\mathrm{PR}=1.11$; 95\%CI: 1.03-1.19) or for whom sputum smear was unperformed $(\mathrm{PR}=1.30 ; 95 \% \mathrm{CI}$ : 1.20-1.40), coinfection ( $\mathrm{PR}=2.04$; CI95\%: 1.89-2.21) and who were not submitted to serology $(\mathrm{PR}=1.62 ; 95 \% \mathrm{CI}: 1.53-1.71)$ have also a higher prevalence of tuberculosis treatment abandonment. Conclusion: Tuberculosis treatment abandonment is associated to biological and social factors, habits, and health service structure.
\end{abstract}

\section{DESCRIPTORS}

Tuberculosis; Treatment Refusal; Epidemiology; Cross-Sectional Studies.

\author{
Autor correspondente: \\ George Jó Bezerra Sousa \\ Universidade Estadual do Ceará, \\ Programa de Pós-graduação Cuidados \\ Clínicos em Enfermagem e Saúde \\ Av. Dr. Silas Munguba, 1700, Itaperi \\ CEP 60714-903 - Fortaleza, CE, Brasil \\ georgejobs@hotmail.com
}

Recebido: 28/08/2020

Aprovado: 19/01/2021 


\section{INTRODUÇÃO}

A tuberculose (TB) é um importante agravo de interesse global. Segundo a Organização Mundial da Saúde (OMS), em 2019 foi estimado que cerca de 10 milhões de pessoas adquiriram a infecção e 1,4 milhão morreram. Além disso, estima-se que $87 \%$ dos casos notificados anualmente são dos 30 países que possuem a maior carga da doença ${ }^{(1)}$.

O Brasil é um dos países que se encontram na lista da OMS que aponta as nações com as maiores taxas de TB e de coinfecção TB-HIV. No país, em 2019, foram registrados 73.834 casos novos de TB, com coeficiente de incidência de 35 casos $/ 100.000$ habitantes. O crescimento da doença foi observado tanto quando foram considerados apenas os municípios com melhores condições socioeconômicas como quando o foco eram os territórios com as piores condições de vida ${ }^{(2)}$.

Dentro do Brasil, o estado do Ceará possui especial relevância, uma vez que, ao longo dos últimos anos, apresenta aumento do número de casos notificados e incidência de 35 casos/100.000 habitantes. Além disso, faz-se necessário estudar a TB no Ceará, pois este é um dos estados brasileiros com taxas de abandono duas vezes maiores que o recomendado pela OMS, que necessita de incremento de políticas de inclusão e proteção de direitos ${ }^{(3)}$.

Diante desse contexto, o abandono do tratamento merece destaque, pois é apontado como fator que influencia o sucesso dos programas de manejo da doença. A OMS preconiza que pelo menos $85 \%$ de todos os casos de TB apresentem a cura como situação de encerramento e, no máximo, $5 \%$ como abandono ${ }^{(4)}$.

Dessa forma, evitar tal desfecho e facilitar a adesão é fundamental mesmo em locais com menor carga de $\mathrm{TB}^{(5)}$. A literatura aponta que o abandono pode se dar por fatores relacionados ao paciente (escolaridade, abuso de drogas, desemprego), aos serviços de saúde (acesso e estrutura) e às características do tratamento em si (tempo de duração e efeitos adversos) $)^{(6-7)}$, sendo um importante obstáculo à cura por elevar o risco de morbidade, mortalidade e resistência medicamentosa ${ }^{(8)}$.

Assim, espera-se que, quando diagnosticadas, as pessoas façam a terapia padrão, que dura cerca de seis meses e, ao seu fim, apresentem o desfecho favorável esperado: a cura. Entretanto, não é o que se observa dentro da prática clínica, visto que muitos indivíduos abandonam e são retratados diversas vezes. Dessa forma, para que sejam criadas estratégias de adesão eficazes, necessita-se saber quais fatores estão mais associados ao desfecho em questão. Portanto, o objetivo deste estudo foi estimar a prevalência de abandono do tratamento da $\mathrm{TB}$ e seus fatores associados.

\section{MÉTODO}

\section{DESENHO DO ESTUdO}

Trata-se de estudo transversal.

\section{Cenário}

Este estudo foi realizado em 2018 em Fortaleza, capital do Ceará, um dos nove estados que compõem a Região Nordeste do Brasil. O Ceará possui a oitava maior população do país (estimada em cerca de 9,13 milhões de habitantes), distribuídos por 184 municípios e densidade demográfica de 56,76 habitantes por $\mathrm{Km}^{2}$ (110 do ranking nacional). Salienta-se que o Ceará é um dos estados mais desiguais do Brasil, pois apresenta Índice de Desenvolvimento Humano de 0,682 ( $17^{\circ}$ do país) e um dos piores rendimentos nominais mensais per capita: cerca de 850 reais $\left(22^{\circ} \text { do país }\right)^{(9)}$.

\section{CRitérios de Seleção}

A fonte de dados utilizada neste estudo foi o Sistema de Informação de Agravos de Notificação (Sinan) para TB. O sistema é formado por um conjunto de variáveis que são obtidas por meio da ficha de notificação e acompanhamento da doença. Essas fichas são preenchidas pelos profissionais de saúde que notificam a doença e são digitadas por profissionais das secretarias de saúde municipal. Os dados compilados de todos os municípios são enviados para as secretarias de saúde estaduais que os consolidam e depois os enviam para o Ministério da Saúde.

A base de dados estadual compreendeu todos os casos notificados de janeiro de 2001 a dezembro de 2017 no estado do Ceará. O critério de inclusão adotado foi o caso ter sido notificado e inserido na base de dados. Em contrapartida, o critério de exclusão foi o não preenchimento da variável desfecho "situação de encerramento". Assim, o estudo contou com uma população de 75.948 notificações; destas, foram excluídas 1.942 por não trazerem informações sobre a situação de encerramento do caso, restando, dessa forma, 74.006 registros na amostra final. Ressalta-se que diversos casos possuíam campos de outras variáveis com dados ausentes, mas eles foram mantidos no estudo, uma vez que outras variáveis poderiam ser utilizadas nas análises.

O desfecho foi definido de acordo com o critério do Ministério da Saúde Brasileiro. Assim, o caso foi notificado como abandono quando o paciente deixava de comparecer ao serviço de saúde por mais de 30 dias consecutivos após a data prevista para o seu retorno. Quando o paciente fazia tratamento supervisionado, consideraram-se 30 dias corridos após a última tomada do medicamento ${ }^{(10)}$.

\section{Coleta de Dados}

Foram coletados dados na Secretaria de Saúde do Estado do Ceará (SESA) provenientes do Sinan-TB em agosto de 2018 pelo pesquisador principal junto com a coordenadoria de vigilância do Estado do Ceará. As variáveis que constavam na ficha de notificação da TB consideradas como possíveis preditoras da doença foram: sexo, idade, escolaridade, raça, zona de moradia, tipo de entrada, forma da doença, realização da baciloscopia de escarro, realização da cultura de escarro, realização dos exames de HIV e aids, alcoolismo e realização do tratamento supervisionado. Outras variáveis 
presentes no banco foram excluídas por apresentarem percentual de dados ausentes acima de 30\%; entretanto, as variáveis "aids" e "alcoolismo" apresentaram dados ausentes acima desse valor, mas foram mantidas devido a sua importância para a epidemiologia da doença e seus desfechos.

Como variável desfecho, escolheu-se a situação de encerramento: abandono do tratamento. Quando necessário, essas variáveis foram transformadas em variáveis dummy para a realização das análises, as quais são variáveis binárias criadas para representar uma variável que originalmente continha múltiplas categorias.

\section{ANÁlISE E TRATAMENTO DOS DADOS}

Para a análise de dados, utilizaram-se frequências simples e relativas para verificar a prevalência de cada variável nominal no desfecho. Contudo, ao se aplicar o teste de ShapiroWilk, verificou-se que a idade não possuía distribuição normal ( $\mathrm{p}<0,001)$, preferindo-se apresentá-la por meio da mediana e intervalo interquartílico (IIQ).

A fim de verificar a associação das variáveis com o desfecho, utilizou-se o teste qui-quadrado e, para a variável numérica, utilizou-se o teste de Mann-Whitney. Em ambos os casos, foram consideradas significantes aquelas que apresentaram $\mathrm{p}<0,05$.

Para calcular a Razão de Prevalência (RP) dos preditores, utilizou-se o modelo de regressão de Poisson com variância robusta. Esse modelo foi escolhido com o objetivo de verificar a estimativa de pontos com maior fidedignidade e intervalos de confiança (IC95\%) mais estreitos. $\mathrm{O}$ modelo multivariado foi ajustado para a idade (contínua), sexo e escolaridade. No modelo inicial, foram inseridas todas as variáveis preditoras que apresentaram $p<0,20$ e, uma a uma, foram removidas aquelas que apresentavam o maior $\mathrm{p}$-valor ${ }^{(11)}$. Assim, permaneceram no modelo final apenas aquelas consideradas estatisticamente significantes $(\mathrm{p}<0,05)$. Todas as análises foram realizadas no software Stata 12.0 (StataCorp LP, College Station, TX, EUA).

\section{Aspectos ÉTICOS}

Por mais que não se tenha contato direto com os pacientes do estudo, a base de dados contém informação individual de cada pessoa notificada. Assim, o projeto foi desenvolvido seguindo os preceitos éticos e legais das Resoluções $n^{\circ}$ 466/12 e no 510/2016 do Conselho Nacional de Saúde (CNS) e foi encaminhado para apreciação pelo Comitê de Ética da Universidade Estadual do Ceará (UECE), tendo sido aprovado sob protocolo de no 2.687.046 de 2018 . Reitera-se que, no momento da coleta da base de dados, o pesquisador, juntamente com o Núcleo de Vigilância da Secretaria de Saúde do Estado do Ceará, removeu quaisquer atributos que identificassem os participantes, como nome, nome da mãe e endereço.

\section{RESULTADOS}

Os 74.006 participantes do estudo apresentaram idade mediana de 38 anos (IQ 26 - 52). A maioria era do sexo masculino $(62,9 \% ; \mathrm{n}=46.537)$, autodeclarada parda $(72,6 \%$; 42.768) e residente na zona urbana $(84,2 \%$; $n=60.235)$. Aproximadamente três em cada quatro indivíduos possuía baixa instrução, pois apresentavam escolaridade igual ou inferior ao ensino fundamental II incompleto (74,08\%; $\mathrm{n}=39.419)$. Entre todos os participantes, foi identificada prevalência de $12,54 \%$ de abandono do tratamento da TB ( $\mathrm{n}=9.280)$. Todas as variáveis sociodemográficas mostraram-se estatisticamente significantes com o abandono $(\mathrm{p}<0,001)($ Tabela 1$)$.

Tabela 1 - Características sociodemográficas de pacientes com abandono do tratamento da tuberculose, 2001-2017 - Fortaleza (CE), Brasil, 2018.

\begin{tabular}{|c|c|c|c|c|}
\hline & \multirow{2}{*}{ Total $(\%)$} & \multicolumn{2}{|c|}{ Abandono } & \multirow{2}{*}{$\mathbf{P}$} \\
\hline & & $\operatorname{Sim}(\%)$ & Não (\%) & \\
\hline Idade (mediana) & $38(26-52)$ & $35(25-45)$ & $38(26-53)$ & $<0,001$ \\
\hline \multicolumn{5}{|l|}{ Sexo $(n=73.984)$} \\
\hline Feminino & $27.447(37,10)$ & $2.822(10,28)$ & $24.625(89,72)$ & $<0,001$ \\
\hline Masculino & $46.537(62,90)$ & $6.457(13,87)$ & $40.080(86,13)$ & \\
\hline Raça/Cor $(n=58.941)$ & & & & $<0,001$ \\
\hline Branca & $10.231(17,36)$ & $864(8,44)$ & $9.367(91,56)$ & \\
\hline Preta & $4.962(8,42)$ & $667(13,44)$ & $4.295(86,56)$ & \\
\hline Amarela & $726(1,23)$ & $88(12,12)$ & $638(87,88)$ & \\
\hline Parda & $42.768(72,56)$ & $5.573(13,03)$ & $37.195(86,97)$ & \\
\hline Indígena & $254(0,43)$ & $22(8,66)$ & $232(91,34)$ & \\
\hline Escolaridade $(n=53.213)$ & & & & $<0,001$ \\
\hline Analfabeto & $9.890(18,59)$ & $1.170(11,83)$ & $8.720(88,17)$ & \\
\hline Fund. I incomp. & $12.411(23,32)$ & $1.478(11,91)$ & $10.933(88,09)$ & \\
\hline
\end{tabular}


...continuação

\begin{tabular}{lccc}
\hline & Total $(\%)$ & Sim $(\%)$ & Não $(\%)$ \\
\cline { 3 - 4 } & & $491(13,35)$ & $3.188(86,65)$ \\
\hline Fund. I comp. & $3.679(6,91)$ & $1.818(13,53)$ & $11.621(86,47)$ \\
Fund. II incomp. & $13.439(25,26)$ & $376(12,29)$ & $2.684(87,71)$ \\
Fund. Il comp. & $3.060(5,75)$ & $495(9,69)$ & $4.615(90,31)$ \\
Ens médio incomp. & $5.110(9,60)$ & $283(7,70)$ & $3.390(92,30)$ \\
Ens médio comp. & $3.673(6,90)$ & $39(7,57)$ & $476(92,43)$ \\
Ens superior incomp. & $515(0,97)$ & $81(5,64)$ & $1.355(94,36)$ \\
Ens superior comp. & $1.436(2,70)$ & & $51.799(85,99)$ \\
Zona de moradia $(\mathbf{n}=71.536)$ & & $8.436(14,01)$ & $10.298(95,12)$ \\
Urbana & $60.235(84,20)$ & $528(4,88)$ & $444(93,47)$ \\
Rural & $10.826(15,14)$ & $31(6,53)$ & 0,001 \\
Periurbana & $475(0,66)$ & & \\
\hline
\end{tabular}

Em relação às características clínico-epidemiológicas, na Tabela 2 é possível identificar que os casos novos representaram cerca de $82 \%$ das notificações $(n=60.614)$, sendo a forma pulmonar a mais prevalente $(82,7 \% ; \mathrm{n}=64.524)$. Quanto aos exames realizados, 5,3\% da amostra apresentou exame positivo para o HIV e, em parcela expressiva dos indivíduos, a baciloscopia de escarro se mostrou positiva $(58,8 \%$; $\mathrm{n}=43.539)$ e o critério de cultura não se aplicou $(81,1 \%$; $\mathrm{n}=60.043)$. Os agravos Aids e alcoolismo foram observados em $8,4 \%(n=3.428)$ e $20,2 \%(n=9.414)$ da população estudada, respectivamente, e o tratamento supervisionado foi realizado por pouco mais da metade dos casos notificados $(55,3 \% ; n=33.630)$. Ressalta-se que assim como as variáveis sociodemográficas, todas as variáveis clínico-epidemiológicas também se mostraram estatisticamente significantes com o abandono do tratamento $(\mathrm{p}<0,001)$.

Tabela 2 - Características clínico-laboratoriais de pacientes com abandono do tratamento da tuberculose, 2001-2017 - Fortaleza (CE), Brasil, 2018.

\begin{tabular}{|c|c|c|c|c|}
\hline & \multirow{2}{*}{ Total } & \multicolumn{2}{|c|}{ Abandono } & \multirow{2}{*}{$\mathbf{P}$} \\
\hline & & $\operatorname{Sim}(\%)$ & Não (\%) & \\
\hline Tipo de entrada $(n=73.944)$ & & & & $<0,001$ \\
\hline Caso novo & $60.614(81,97)$ & $6.402(10,56)$ & $54.212(89,44)$ & \\
\hline Recidiva & $5.028(6,80)$ & $728(14,48)$ & $4.300(85,52)$ & \\
\hline Reingresso pós-abandono & $4.302(5,82)$ & $1.712(39,80)$ & $2.590(60,20)$ & \\
\hline Não sabe & $843(1,14)$ & $164(19,45)$ & $679(80,55)$ & \\
\hline Transferência & $3.157(4,27)$ & $273(8,65)$ & $2.884(91,35)$ & \\
\hline Forma da doença $(n=73.988)$ & & & & $<0,001$ \\
\hline Pulmonar & $64.524(87,21)$ & $8.265(12,81)$ & $56.259(87,19)$ & \\
\hline Extrapulmonar & $8.192(11,07)$ & $827(10,10)$ & $7.365(89,90)$ & \\
\hline Mista & $1.272(1,72)$ & $187(14,70)$ & $1.085(85,30)$ & \\
\hline \multicolumn{5}{|c|}{ Baciloscopia de escarro $(n=73.988)$} \\
\hline Positiva & $43.539(58,84)$ & $5.410(12,43)$ & $38.129(87,57)$ & $<0,001$ \\
\hline Negativa & $14.495(19,59)$ & $1.398(9,64)$ & $13.097(90,36)$ & \\
\hline Não realizada & $15.364(20,77)$ & $2.368(15,41)$ & $12.996(84,59)$ & \\
\hline Não se aplica & $590(0,80)$ & $103(17,46)$ & $487(82,54)$ & \\
\hline \multicolumn{5}{|l|}{ Cultura de escarro $(n=73.988)$} \\
\hline Positiva & $7.443(10,06)$ & $972(13,06)$ & $6.471(86,94)$ & $<0,001$ \\
\hline Negativa & $4.036(5,45)$ & $283(7,01)$ & $3.753(92,99)$ & \\
\hline Não realizada & $2.466(3,34)$ & $255(10,34)$ & $2.211(89,66)$ & \\
\hline
\end{tabular}

continua... 


\begin{tabular}{|c|c|c|c|c|}
\hline & \multirow{2}{*}{ Total } & \multicolumn{2}{|c|}{ Abandono } & \multirow{2}{*}{$\mathbf{P}$} \\
\hline & & $\operatorname{Sim}(\%)$ & Não (\%) & \\
\hline Não se aplica & $60.043(81,15)$ & $7.769(12,94)$ & $52.274(87,06)$ & \\
\hline HIV $(n=74.005)$ & & & & $<0,001$ \\
\hline Positivo & $3.953(5,34)$ & $894(22,62)$ & $3.059(77,38)$ & \\
\hline Negativo & $26.233(35,45)$ & $2.447(9,33)$ & $23.786(90,67)$ & \\
\hline Em andamento & $2.549(3,44)$ & $230(9,02)$ & $2.319(90,98)$ & \\
\hline Não realizado & $41.270(55,77)$ & $5.709(13,83)$ & $35.561(86,17)$ & \\
\hline Aids $(n=40.787)$ & & & & $<0,001$ \\
\hline Sim & $3.428(8,40)$ & $786(22,93)$ & $2.642(77,07)$ & \\
\hline Não & $37.359(91,60)$ & $4.505(12,06)$ & $32.854(87,94)$ & \\
\hline Alcoolismo $(n=46.531)$ & & & & $<0,001$ \\
\hline Sim & $9.414(20,23)$ & $1.893(20,11)$ & $7.521(79,89)$ & \\
\hline Não & $37.117(79,77)$ & $4.234(11,41)$ & $32.883(88,59)$ & \\
\hline Tratamento supervisionado $(n=54.789)$ & & & & $<0,001$ \\
\hline Sim & $33.630(55,32)$ & $3.976(11,82)$ & $29.654(88,18)$ & \\
\hline Não & $10.044(26,39)$ & $2.096(13,06)$ & $13.948(86,94)$ & \\
\hline Ignorado & $11.115(18,29)$ & $1.268(11,41)$ & $9.847(88,59)$ & \\
\hline
\end{tabular}

Como todas as variáveis se mostraram estatisticamente significantes nos testes bivariados, elas foram inseridas no modelo multivariado que, por sua vez, foi ajustado para a idade (contínua), o sexo e a escolaridade. As variáveis não significantes do modelo inicial foram removidas uma a uma até se chegar ao modelo composto apenas pelas significantes. No modelo final, observou-se que morar em zona urbana aumenta a prevalência de abandono do tratamento em 2,45 vezes (IC95\%: 2,20 - 2,74) em relação à zona rural. Além disso, identificou-se que, quando comparados aos casos novos, aqueles em recidiva apresentam prevalência de abandono aumentada em $22 \%$ (IC95\%: 1,10 - 1,35) e em 2,84 vezes entre aqueles classificados como reingresso pós abandono $(2,68-3,01)$. Em contrapartida, quando o caso é notificado como transferência, o abandono reduz em 20\% (IC95\%: 0,69 - 0,92) (Tabela 3).

Não obstante, a forma extrapulmonar da doença reduz em 20\% a prevalência do desfecho (IC95\%: 0,73-0,88). Em relação à baciloscopia de escarro negativa, a baciloscopia positiva e a não realizada aumentaram o abandono em 11\% (IC95\%: 1,03 - 1,19) e 30\% (IC95\%: 1,20 - 1,40), respectivamente. Houve também um aumento de cerca de duas vezes na prevalência do abandono quando o exame de HIV se mostrava positivo (IC95\%: 1,89-2,21) e de 77\% quando ele não era realizado (IC95\%: 1,64-1,90). Por fim, o alcoolismo aumentou em $50 \%$ o abandono do tratamento $(1,42-1,58)$ (Tabela 3$)$.

Tabela 3 - Modelo de regressão de Poisson robusta ajustada para idade (contínua), sexo e escolaridade para estimar as razões de prevalência dos fatores associados ao abandono do tratamento, 2001-2017 - Fortaleza (CE), Brasil, 2018.

\begin{tabular}{|c|c|c|c|c|}
\hline & Modelo inicial & \multirow{2}{*}{$\mathbf{P}$} & Modelo final & \multirow{2}{*}{$\mathbf{P}$} \\
\hline & RP (IC95\%) & & RPA (IC95\%) & \\
\hline \multicolumn{5}{|l|}{ Zona de moradia } \\
\hline Rural & 1 & - & 1 & - \\
\hline Urbana & $2,73(2,34-3,18)$ & $<0,001$ & $2,45(2,20-2,74)$ & $<0,001$ \\
\hline Periurbana & $1,66(0,96-2,88)$ & 0,070 & & \\
\hline \multicolumn{5}{|l|}{ Tipo de entrada } \\
\hline Caso novo & 1 & - & 1 & - \\
\hline Recidiva & $1,36(0,99-1,30)$ & 0,061 & $1,22(1,10-1,35)$ & $<0,001$ \\
\hline Reingresso pós-abandono & $2,71(2,51-2,93)$ & $<0,001$ & $2,84(2,68-3,01)$ & $<0,001$ \\
\hline Transferência & $0,72(0,59-0,87)$ & 0,001 & $0,80(0,69-0,92)$ & 0,002 \\
\hline
\end{tabular}


...continuação

\begin{tabular}{|c|c|c|c|c|}
\hline & Modelo inicial & \multirow{2}{*}{$\mathbf{P}$} & Modelo final & \multirow{2}{*}{$\mathbf{P}$} \\
\hline & RP (IC95\%) & & RPA (IC95\%) & \\
\hline \multicolumn{5}{|l|}{ Forma da doença } \\
\hline Pulmonar & 1 & - & 1 & - \\
\hline Extrapulmonar & $0,81(0,72-0,92)$ & 0,001 & $0,80(0,73-0,88)$ & $<0,001$ \\
\hline Mista & $1,09(0,89-1,33)$ & 0,393 & & \\
\hline \multicolumn{5}{|c|}{ Baciloscopia de escarro } \\
\hline Negativa & 1 & - & 1 & - \\
\hline Positiva & $1,15(1,05-1,26)$ & 0,002 & $1,11(1,03-1,19)$ & 0,004 \\
\hline Não realizada & $1,28(1,15-1,42)$ & $<0,001$ & $1,30(1,20-1,40)$ & $<0,001$ \\
\hline \multicolumn{5}{|l|}{ Cultura de escarro } \\
\hline Negativa & 1 & - & & \\
\hline Positiva & $1,04(0,94-1,15)$ & 0,467 & & \\
\hline Não realizada & $1,04(0,88-1,24)$ & 0,642 & & \\
\hline \multicolumn{5}{|l|}{ HIV } \\
\hline Negativo & 1 & - & 1 & - \\
\hline Positivo & $2,26(1,74-2,93)$ & $<0,001$ & $2,04(1,89-2,21)$ & $<0,001$ \\
\hline Em andamento & $1,14(0,92-1,42)$ & 0,242 & & \\
\hline Não realizado & $1,77(1,64-1,90)$ & $<0,001$ & $1,62(1,53-1,71)$ & $<0,001$ \\
\hline \multicolumn{5}{|l|}{ Aids } \\
\hline Não & 1 & - & & \\
\hline Sim & $1,03(0,79-1,35)$ & 0,838 & & \\
\hline \multicolumn{5}{|l|}{ Alcoolismo } \\
\hline Não & 1 & - & 1 & - \\
\hline $\operatorname{Sim}$ & $1,62(1,50-1,75)$ & $<0,001$ & $1,50(1,42-1,58)$ & $<0,001$ \\
\hline \multicolumn{5}{|c|}{ Tratamento supervisionado } \\
\hline Não & 1 & - & & \\
\hline Sim & $0,95(0,88-1,03)$ & 0,194 & & \\
\hline Ignorado & $1,00(0,83-1,21)$ & 0,963 & & \\
\hline
\end{tabular}

\section{DISCUSSÃO}

Neste estudo identificou-se elevada prevalência de abandono do tratamento da TB no estado do Ceará, Brasil. Evidenciou-se que viver em zona urbana, dar entrada como reingresso pós-abandono ou recidiva e ser HIV-positivo são fatores fortemente associados a esse desfecho. O mesmo padrão foi observado quando os exames diagnósticos da TB e HIV não são realizados. Não obstante, o alcoolismo mostrou-se um importante fator na cadeia causal do abandono do tratamento da doença. Em contrapartida, o modelo multivariado sugeriu redução na prevalência da TB quando o caso foi notificado como transferência e a doença havia se manifestado na forma extrapulmonar.

A urbanização é um dos fenômenos considerados na cadeia de desfechos da TB, pois quando examinada a localização geográfica, devem ser observadas em maiores detalhes as regiões mais populosas e o acesso aos serviços de saúde ${ }^{(12)}$. Embora este estudo tenha identificado que viver em zonas urbanas aumenta a prevalência de abandono do tratamento $\mathrm{da} \mathrm{TB}$, a literatura diverge acerca do quanto a zona de moradia pode influenciar no abandono do tratamento da doença. Enquanto estudo realizado na Nigéria ${ }^{(12)}$ identificou que viver em zona rural eleva as chances de desfechos negativos da TB (óbito, abandono e falência), investigação realizada no Brasil ${ }^{(13)}$ não identificou diferenças significativas entre os locais de moradia.

Em relação ao tipo de entrada, foi identificado que a recidiva e, especialmente, o reingresso pós-abandono podem colaborar para um novo abandono. A literatura endossa esses achados com estudos conduzidos na África do Sul ${ }^{(14)}, \mathrm{Brasi}^{(15)}$ e Etiópia ${ }^{(16)}$. Nesse sentido, é fundamental que estratégias de adesão sejam realizadas logo no primeiro tratamento, a fim de se obter um desfecho favorável. Diversas são as possibilidades que podem ser usadas para evitar um novo abandono. Entre elas estão o tratamento diretamente observado (TDO), que ajuda os pacientes na tomada regular de medicamentos, bem como alternativas ao TDO, como o observado por vídeo ${ }^{(17-18)}$. Outro aspecto importante é a participação ativa da equipe de saúde, assim como da família ${ }^{(19-20)}$. 
A forma extrapulmonar foi identificada nesta pesquisa como fator de decréscimo do abandono do tratamento. Corroborando esses achados, estudos ${ }^{(21-23)}$ também identificaram a diminuição do abandono do tratamento quando as formas não pulmonares são manifestadas. Por outro lado, estudo realizado no Paquistão ${ }^{(24)}$ não encontrou associações significantes e investigação desenvolvida na Malásia ${ }^{(25)}$ apontou aumento dos desfechos desfavoráveis quando a manifestação é extrapulmonar. Entretanto, deve-se ressaltar que, além do abandono, a categoria desfavorável contemplou outros desfechos, como o óbito, a transferência, a falência e a não avaliação. Portanto, esses resultados devem ser interpretados com cautela.

Outro fator de fundamental importância no abandono do tratamento da TB é a sua coinfecção com o HIV, tendo em vista a evidência de aumento em cerca de duas vezes a prevalência desse desfecho quando se observa a coinfecção. Semelhante ao verificado nessa investigação, pesquisas realizadas em todo o globo apontam aumento nas chances de abandono do tratamento quando há coinfecção entre as duas doenças ${ }^{(12,21-23)}$.

O Brasil é um dos países que compõem a lista da OMS com o maior número de casos de coinfecção TB-HIV ${ }^{(1)}$. Isso se torna ainda mais relevante quando se estima que de 2002 a 2012 houve aumento de $9,52 \%$ para $13,60 \%$ no abandono do tratamento em pessoas com TB-HIV ${ }^{(26)}$. Assim, a não adesão ao tratamento de pessoas com coinfecção TB-HIV pode ser devida à necessidade do uso de terapêutica adicional que, por conseguinte, pode gerar possíveis efeitos adversos. Além disso, a literatura também levanta a hipótese de que a não adesão também pode ser reflexo da falta de vínculo com os profissionais de saúde, os quais, além das questões físicas, devem também buscar entender as questões psicológicas desses pacientes ${ }^{(27)}$.

Ademais, evidenciou-se que a não realização de testes diagnósticos tanto da TB quanto do HIV podem influenciar negativamente no tratamento. Esse importante alerta se dá uma vez que nesta investigação estimou-se aumento na prevalência do abandono quando o status do HIV e da TB são desconhecidos. Assim, de forma similar ao encontrado nesta investigação, pesquisas também apontam aumento no abandono quando o status de HIV é desconhecido ${ }^{(14,28)}$. Esse dado mostra fragilidade nos serviços de saúde e na atenção às doenças infecciosas. Em face disso, evidencia-se a necessidade de intensificação nas políticas de testagem e acompanhamento dos casos, bem como a importância de conhecer o estado sorológico para o HIV dos indivíduos diagnosticados com TB para que o tratamento ocorra de forma mais segura.

Por fim, ressalta-se que a drogadição é fator essencial na cadeia causal do abandono do tratamento da TB, tendo em vista que esta pesquisa verificou aumento na prevalência de abandono quando a pessoa era alcoolista. Estudos realizados no Peru ${ }^{(28)}$ e no Brasil ${ }^{(23)}$ apontam acréscimo nesse desfecho quando a pessoa utiliza álcool ou outras drogas. Desse modo, entende-se que a identificação precoce do uso de álcool e outras drogas pode levar à adesão ao tratamento se a equipe de saúde for devidamente treinada para identificar e implementar o TDO nessa população ${ }^{(29)}$.

A principal limitação deste estudo se refere ao uso de base de dados secundários guiada pela ficha de notificação da TB, que mudou no ano de 2015. Essa mudança impossibilitou a utilização de diversas variáveis devido a seu elevado grau de incompletude. Entretanto, as variáveis selecionadas e as análises utilizadas foram escolhidas para superar esse obstáculo.

\section{CONCLUSÃO}

Neste estudo, observou-se que o abandono do tratamento da TB está associado a diversos fatores, sendo eles referentes à moradia (zona urbana), à doença (tipo de entrada e forma da doença), aos serviços de saúde (não realização de baciloscopia e do exame de HIV) e ao seu estado de saúde (HIV positivo, alcoolismo). Dessa forma, tais características podem subsidiar intervenções com o intuito de melhorar os desfechos da doença.

Assim, é necessário entender que o desfecho investigado vai além dos fatores aqui expostos, uma vez que ele é complexo e multifatorial. Ao entender essa cadeia de fatores, gestores e profissionais de saúde podem reconhecer perfil epidemiológico de uma parcela da população do Nordeste brasileiro que merece foco de intervenções para fortalecimento da adesão ao tratamento da TB. Portanto, deve-se notar a importância de políticas públicas a nível local e nacional a fim de modificar a cadeia de transmissão da doença e erradicar o abandono do tratamento da doença.

\section{RESUMO}

Objetivo: Estimar a prevalência de abandono do tratamento da tuberculose e seus fatores associados. Método: Estudo transversal que utilizou os casos de tuberculose do Sistema de Informação de Agravos de Notificação entre 2001 e 2017 no Ceará. Fizeram parte desse estudo 74.006 casos e o desfecho foi a situação de encerramento "abandono do tratamento". Foi realizada análise multivariada para estimar associação entre as variáveis com o abandono. Resultados: Durante todo o período, a taxa de abandono foi de $12,54 \%$. Verificou-se maior prevalência de abandono entre as pessoas que residem na zona urbana ( $R P=2,45 ; \mathrm{IC} 95 \%: 2,20-2,74)$, que dão entrada como reingresso pós-abandono $(\mathrm{RP}=2,84$; IC95\%: 2,68-3,01), entre aqueles notificados como recidiva $(\mathrm{RP}=1,22 ; \mathrm{IC} 95 \%$ : $1,10-1,35)$ e entre os etilistas $(R P=1,50 ; \mathrm{IC} 95 \%: 1,42-1,58)$. Aqueles que apresentaram baciloscopia de escarro positiva $(\mathrm{RP}=1,11$; IC95\%: 1,03-1,19) ou não realizada $(R P=1,30$; IC95\%: 1,20-1,40), coinfecção $(R P=2,04 ;$ IC95\%: $1,89-2,21)$ e que não realizaram sorologia ( $\mathrm{RP}=1,62 ; \mathrm{IC} 95 \%: 1,53-1,71)$ também possuem maior prevalência de abandono do tratamento para tuberculose. Conclusão: $\mathrm{O}$ abandono do tratamento da tuberculose está associado a fatores biológicos, sociais, hábitos e à estrutura dos serviços de saúde.

\section{DESCRITORES}

Tuberculose; Recusa do Paciente ao Tratamento; Epidemiologia; Estudos Transversais.

\section{RESUMEN}

Objetivo: Estimar la prevalencia del abandono del tratamiento de la tuberculosis y sus factores asociados. Método: Estudio transversal que utilizó los casos de tuberculosis del Sistema de Información de Agravios de Notificación entre 2001 y 2017 en Ceará, Brasil. 
Un total de 74.006 casos fueron incluidos en este estudio y el resultado fue la conclusión "abandono del tratamiento". Se realizó un análisis multivariante para estimar la asociación entre las variables con el abandono. Resultados: Durante todo el periodo, la tasa de abandono fue del $12,54 \%$. Se encontró una mayor prevalencia de abandono entre las personas que viven en zonas urbanas $(\mathrm{RP}=2,45$; IC95\%: 2,20-2,74), los que ingresan como reingreso tras el abandono ( $\mathrm{RP}=2,84$; IC95\%: 2,68-3,01), los que se declararon reincidentes $(\mathrm{RP}=1,22$; IC95\%: 1,10-1,35) y los alcohólicos ( $\mathrm{RP}=1,50$; IC95\%: 1,42-1,58). Los que tenían una baciloscopia de esputo positiva $(\mathrm{RP}=1,11$; IC95\%: 1,03-1,19) o para los cuales no se realizó baciloscopia de esputo $(\mathrm{RP}=1,30$; IC95\%: 1,20-1,40), coinfección $(R P=2,04$; IC95\%: 1,89-2,21) y que no se sometieron a serología $(R P=1,62 ;$ IC95\%: 1,53-1,71) también presentaron una mayor prevalencia de abandono del tratamiento de la tuberculosis. Conclusión: El abandono del tratamiento de la tuberculosis está asociado a factores biológicos y sociales, a los hábitos y a la estructura de los servicios sanitarios.

\section{DESCRIPTORES}

Tuberculosis; Negativa del Paciente al Tratamiento; Epidemiología; Estudios Transversales.

\section{REFERÊNCIAS}

1. World Health Organization. Global tuberculosis report 2020 [Internet]. Geneva: World Health Organization; 2020 [cited 2020 Dec 7]. Available from: https://www.who.int/teams/global-tuberculosis-programme/tb-reports

2. Brasil. Ministério da Saúde. Boletim Epidemiológico. Tuberculose 2020 [Internet]. Brasília, DF: Ministério da Saúde; 2020 [cited 2020 Dec 7]. Available from: https://antigo.saude.gov.br/images/pdf/2020/marco/24/Boletim-tuberculose-2020-marcas--1-.pdf

3. Ceará. Secretaria de Saúde. Boletim epidemiológico da tuberculose [Internet]. Fortaleza: Secretaria de Saúde; 2020 [cited 2020 Dec 7]. Available from: https://www.saude.ce.gov.br/wp-content/uploads/sites/9/2018/06/boletim_tuberculose_02_10_2020.pdf

4. Beraldo AA, Andrade RL, Orfão NH, Silva-Sobrinho RA, Pinto ES, Wysocki AD, et al. Adherence to tuberculosis treatment in Primary Health Care: perception of patients and professionals in a large municipality. Esc Anna Nery. 2017;21(4):e20170075. https://doi.org/10.1590/21779465-ean-2017-0075

5. Arakawa T, Magnabosco GT, Andrade RL, Brunello ME, Monroe AA, Ruffino-Netto A, et al. Tuberculosis control program in the municipal context: performance evaluation. Rev Saude Publica. 2017;51:23. https://doi.org/10.1590/s1518-8787.2017051006553

6. Lima LM, Harter J, Tomberg JO, Vieira DA, Antunes ML, Cardozo-Gonzales RI. Monitoring and assessment of outcome in cases of tuberculosis in a municipality of Southern Brazil. Rev Gaúcha Enferm. 2016;37(1):e51467. https://doi.org/10.1590/1983-1447.2016.01.51467

7. Furlan MC, Marcon SS. [Evaluation of access to tuberculosis treatment from the point of view of patients]. Cad Saude Colet. 2017;25(3):33947. Portuguese. https://doi.org/10.1590/1414-462×201700030139

8. Putera I, Pakasi TA, Karyadi E. Knowledge and perception of tuberculosis and the risk to become treatment default among newly diagnosed pulmonary tuberculosis patients treated in primary health care, East Nusa Tenggara: a retrospective study. BMC Res Notes. 2015;8(1):238. https://doi.org/10.1186/s13104-015-1209-6

9. Instituto Brasileiro de Geografia e Estatística. Brasil - Ceará [Internet]. Rio de Janerio: Instituto Brasileiro de Geografia e Estatística; 2020 [cited 2020Jun 12]. Available from: https://cidades.ibge.gov.br/brasil/ce/panorama

10. Brasil. Ministério da Saúde. Manual de recomendações para o controle da tuberculose no Brasil [Internet]. Brasília, DF: Ministério da Saúde; 2019 [cited 2020 jun.Jun 15]. Available from: http://bvsms.saude.gov.br/bvs/publicacoes/manual_recomendacoes_controle_ tuberculose_brasil_2_ed.pdf

11. Coutinho LM, Scazufca M, Menezes PR. Methods for estimating prevalence ratios in cross-sectional studies. Rev Saude Publica. 2008;42(6):992-8. https://doi.org/10.1590/S0034-89102008000600003

12. Adamu AL, Aliyu MH, Galadanci NA, Musa BM, Lawan UM, Bashir U, et al. The impact of rural residence and HIV infection on poor tuberculosis treatment outcomes in a large urban hospital: a retrospective cohort analysis. Int J Equity Health. 2018;17(1):4. https://doi.org/10.1186/s12939-017-0714-8

13. Basta PC, Marques M, Oliveira RL, Cunha EA, Resendes AP, Souza-Santos R. Social inequalities and tuberculosis: an analysis by race/color in Mato Grosso do Sul, Brazil. Rev Saude Publica. 2013;47(5):854-64. https://doi.org/10.1590/S0034-8910.2013047004628

14. Kigozi G, Heunis C, Chikobvu P, Botha S, Rensburg D. Factors influencing treatment default among tuberculosis patients in a high burden province of South Africa. Int J Infect Dis. 2017;54:95-102. https://doi.org/10.1016/j.ijid.2016.11.407

15. Silva EA, Anjos UU, Nogueira JA. [Predictive model to the tuberculosis treatment abandonment]. Saúde Debate. 2014;38(101):200-9. Portuguese. https://doi.org/10.5935/0103-1104.20140018

16. Tesfahuneygn G, Medhin G, Legesse M. Adherence to Anti-tuberculosis treatment and treatment outcomes among tuberculosis patients in Alamata District, northeast Ethiopia. BMC Res Notes. 2015;8(1):503. https://doi.org/10.1186/s13104-015-1452-x

17. Alipanah N, Jarlsberg L, Miller C, Linh NN, Falzon D, Jaramillo E, et al. Adherence interventions and outcomes of tuberculosis treatment: a systematic review and meta-analysis of trials and observational studies. PLoS Med. 2018;15(7):e1002595. https://doi.org/10.1371/ journal.pmed.1002595

18. Müller AM, Osório CS, Silva DR, Sbruzzi G, de Tarso P, Dalcin R. Interventions to improve adherence to tuberculosis treatment: systematic review and meta-analysis. Int J Tuberc Lung Dis. 2018;22(7):731-40. https://doi.org/10.5588/ijtld.17.0596

19. Oliveira RA, Lefèvre F. Communication on disclosure of tuberculosis diagnosis and adherence to treatment: social representations of professionals and patients. Texto Contexto Enferm. 2017;26(2):e06790015. https://doi.org/10.1590/0104-07072017006790015

20. Chirinos NE, Meirelles BH, Bousfield ABS. Social representations of TB patients on treatment discontinuation. Rev Gaúcha Enferm. 2015;36(spe):207-14. https://doi.org/10.1590/1983-1447.2015.esp.56723

21. Harling G, Lima Neto AS, Sousa GS, Machado MM, Castro MC. Determinants of tuberculosis transmission and treatment abandonment in Fortaleza, Brazil. BMC Public Health. 2017;17(1):508. https://doi.org/10.1186/s12889-017-4435-0 
22. Snyder RE, Marlow MA, Phuphanich ME, Riley LW, Maciel EL. Risk factors for differential outcome following directly observed treatment (DOT) of slum and non-slum tuberculosis patients: a retrospective cohort study. BMC Infect Dis. 2016;16(1):494. https://doi.org/10.1186/ s12879-016-1835-1

23. Prado TN, Rajan JV, Miranda AE, Dias ED, Cosme LB, Possuelo LG, et al. Clinical and epidemiological characteristics associated with unfavorable tuberculosis treatment outcomes in TB-HIV co-infected patients in Brazil: a hierarchical polytomous analysis. Braz J Infect Dis. 2017;21(2):162-70. https://doi.org/10.1016/j.bjid.2016.11.006

24. Chida N, Ansari Z, Hussain H, Jaswal M, Symes S, Khan AJ, et al. Determinants of default from tuberculosis treatment among patients with drug-susceptible tuberculosis in Karachi, Pakistan: a mixed methods study. PLoS One. 2015;10(11):e0142384. https://doi.org/10.1371/ journal.pone.0142384

25. Liew SM, Khoo EM, Ho BK, Lee YK, Mimi O, Fazlina MY, et al. Tuberculosis in Malaysia: predictors of treatment outcomes in a national registry. Int J Tuberc Lung Dis. 2015;19(7):764-71. https://doi.org/10.5588/ijtld.14.0767

26. Gaspar RS, Nunes N, Nunes M, Rodrigues VP. Temporal analysis of reported cases of tuberculosis and of tuberculosis-HIV co-infection in Brazil between 2002 and 2012. J Bras Pneumol. 2016;42(6):416-22. https://doi.org/10.1590/s1806-37562016000000054

27. Magnabosco GT, Andrade RL, Arakawa T, Monroe AA, Villa TC. Tuberculosis cases outcome in people with HIV: intervention subsidies. Acta Paul Enferm. 2019;32(5):554-63. https://doi.org/10.1590/1982-0194201900077

28. Lackey B, Seas C, Van der Stuyft P, Otero L. Patient characteristics associated with tuberculosis treatment default: a cohort study in a high-incidence area of Lima, Peru. PLoS One. 2015;10(6):e0128541. https://doi.org/10.1371/journal.pone.0128541

29. Silva MR, Pereira JC, Costa RR, Dias JA, Guimarães MD, Leite IC. Drug addiction and alcoholism as predictors for tuberculosis treatment default in Brazil: a prospective cohort study. Epidemiol Infect. 2017;145(16):3516-24. https://doi.org/10.1017/S0950268817002631 modalidade bolsa de mestrado processo BMD-0008-01031.01.06/17. 